\title{
High-precision Difiraction Grating and Large-aperture SiC Mirror
}

A diffraction grating is the tool of choice for separating the colors in incident light. It is the -heart" of various spectroscopic instruments. It has important applications in the fields of astronomy. optical communications, lasers, information storage. inertial confinement fusion, etc. It is a worldwide challenge to make the grating larger and finer because of the diffraction grating ruling engines used to fabricate gratings are known as "the king of fine mechanics".

After vears of efforts. scientists at the CAS Changchun Institute of Optics, Fine Mechanics and Physics (CIOMP) made a series of key technical breakthroughs. In November 2016, they independently developed a large-scale high-precision diffraction grating ruling system, and successfully produced the world's largest echelle gratings (400 mm x $500 \mathrm{~mm}$ ), providing the "hearts" for China's spectrum instruments. This achievement has broken a long-lasting technical barrier. enabling China to enter the high-tech end of the spectrum instrument industry and international market.

The CIONP scientists also made many breakthroughs in the field of large-aperture optical mirror. large-aperture optical mirror is the core element for high-resolution space-to-ground observation. deep space exploration and astronomical observation systems. Silicon carbide (SiC) mirror is a catch with global acknowledgement.

In 2018, scientists at the CIOMP developed the world's largest $\mathrm{SiC}$ mirror - a high-precision $\mathrm{SiC}$ aspherical mirror with a diameter of 4.03 meters. These state-of-the-art $\mathrm{SiC}$ mirrors can be widely used in astronomical telescopes and spacecrafts.

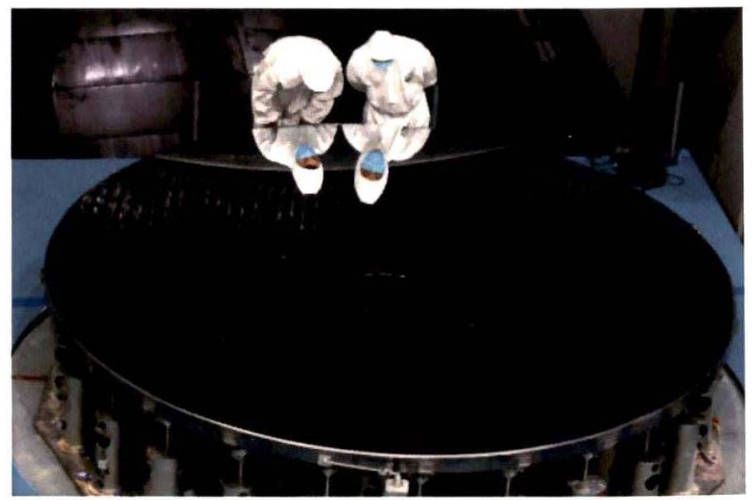

Scientists were carefully examining the SiC mirror. (Credit: CIOMP)
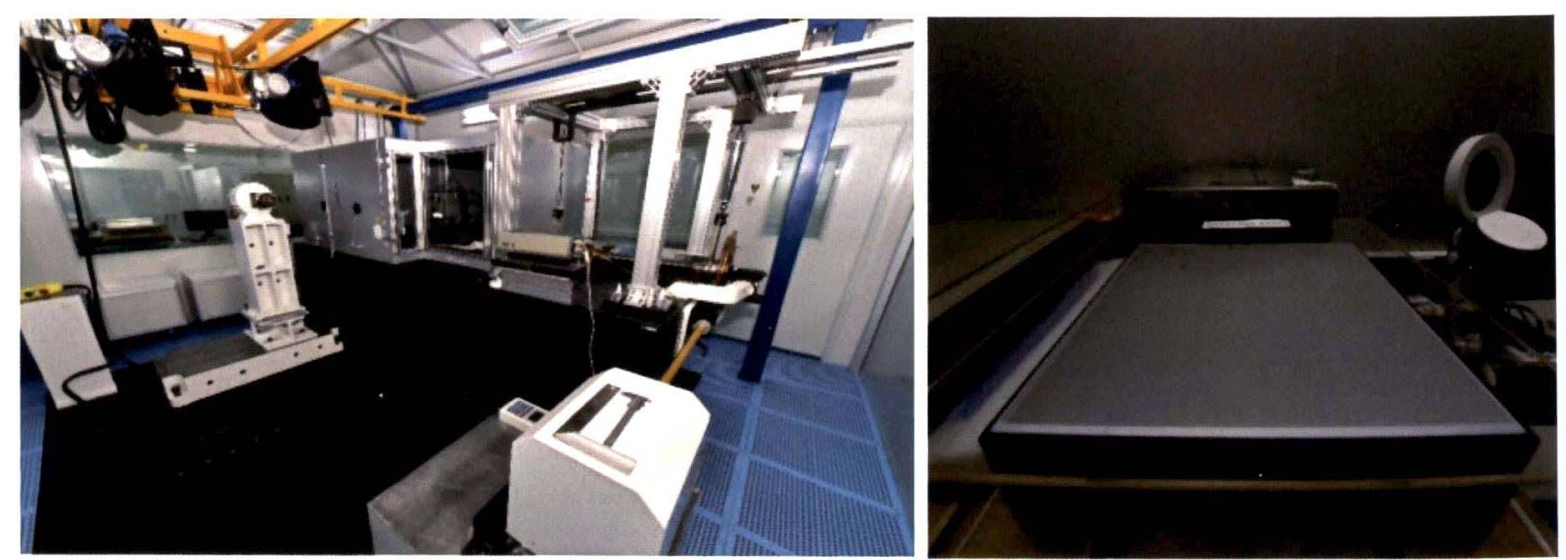

Left: A large-scale high-precision diffraction grating ruling system. Right: A world's largest $400 \mathrm{~mm} \times 500 \mathrm{~mm}$ echelle grating. (Credit: ClOMP) 